\title{
Graph Classification on Dissimilarity Space Embedding
}

\author{
Horst Bunke and Kaspar Riesen
}

\begin{abstract}
Recently, an emerging trend of representing objects by graphs can be observed. In fact, graphs offer a powerful alternative to feature vectors in pattern recognition, machine learning, and related fields. However, the domain of graphs contains very little mathematical structure, and consequently, there is only a limited amount of classification algorithms available. In this paper we survey recent work on graph embedding using dissimilarity representations. Once a population of graphs has been mapped to a vector space by means of this embedding procedure, all classification methods developed in statistical pattern recognition become directly available. In an experimental evaluation we show that the proposed methodology of first embedding graphs in vector spaces and then applying a statistical classifier has significant potential to outperform classifiers that directly operate in the graph domain. Additionally, the proposed framework can be considered a contribution towards unifying the domains of structural and statistical pattern recognition.
\end{abstract}

